\title{
Optimal replacement policies for dairy cows based on daily yield measurements
}

\author{
L. R. Nielsen, ${ }^{* 1}$ E. Jørgensen,† A. R. Kristensen,‡ and S. Østergaard§ \\ *Department of Business Studies, Aarhus University, Fuglesangs Allé 4, DK-8210 Aarhus V, Denmark \\ †Department of Genetics and Biotechnology, Aarhus University, PO Box 50, DK-8830 Tjele, Denmark \\ ‡Department of Large Animal Sciences, University of Copenhagen, Grønnegårdsvej 2, DK-1870 Frederiksberg C, Denmark \\ $\S$ Department of Animal Health and Bioscience, Aarhus University, PO Box 50, DK-8830 Tjele, Denmark
}

\section{ABSTRACT}

Markov decision processes (MDP) with finite state and action space have often been used to model sequential decision making over time in dairy herds. However, the length of each stage has been at least 1 mo, resulting in models that do not support decisions on a daily basis. The present paper describes the first step of developing an MDP model that can be integrated into a modern herd management system. A hierarchical MDP was formulated for the dairy cow replacement problem with stage lengths of $1 \mathrm{~d}$. It can be used to assist the farmer in replacement decisions on a daily basis and is based on daily milk yield measurements that are available in modern milking systems. Bayesian updating was used to predict the performance of each cow in the herd and economic decisions were based on the prediction. Moreover, parameters in the model were estimated using data records of the specific herd under consideration. This includes herd-specific lactation curves.

Key words: hierarchical Markov decision process, stochastic dynamic programming, state space model, herd management

\section{INTRODUCTION}

The decision of when to replace a cow with a heifer is affected by many factors such as the cow's current and future milk yield, illness, availability of replacement heifers, prices, reproduction, and the producer's goals (see, for example, Bascom and Young, 1998 and Monti et al., 1999). The problem is sequential in nature: at a specific time the decision of whether to replace the cow or not is based on known information and expectations about the future. At the next decision stage, updated information is available and the decision choice is reevaluated.

Received March 13, 2009.

Accepted September 1, 2009.

${ }^{1}$ Corresponding author: lars@relund.dk
A sequential decision making problem can be solved using a Markov decision process (MDP). At a specified point in time, the decision maker observes the state of a system and makes a decision. The decision and the state of the process produce 2 results: the decision maker receives an immediate reward (or incurs an immediate cost), and the system evolves probabilistically to a new state at a subsequent discrete point in time. At this subsequent point in time, the decision maker faces a similar problem. However, the observed state may be different from the previous observed state. The goal is to find a policy of decisions (dependent on the observation of the state) that maximizes, for example, the expected discounted reward.

In the dairy industry, MDP have been used over the past several decades to calculate optimal replacement policies. In the early 1960s, Jenkins and Halter (1963) suggested the use of an MDP in a simple dairy cow replacement example. A few years later, Giaever (1966) presented a detailed description of the dairy cow replacement problem and how it can be solved using an MDP.

Kristensen (1987) suggested modeling the dairy cow replacement problem using a hierarchical Markov decision process (HMDP). The model is a series of MDP built together into one MDP called the main process. As a result, the age of the cow can be omitted in the state space compared with an ordinary MDP model. Moreover, it takes into account that the production is cyclic. When a replacement occurs, not just a regular state transition takes place but rather the process (life cycle of the replacement animal) is restarted. By using HMDP, more detailed models can be solved; however, only a few studies have used this method on the dairy cow replacement problem (Kristensen, 1987, 1989; Houben et al., 1994). Later, Kristensen and Jørgensen (2000) extended the methodology to multilevel HMDP such that MDP can be built together at multiple levels. An HMDP is an infinite-stage MDP with parameters defined in a special way, but nevertheless in accordance with all usual rules and conditions relating to such processes. The basic idea of the hierarchic 
structure is that stages of the process can be expanded to a so-called child process, which again may expand stages further to new child processes leading to multiple levels. Recently, Bar et al. (2008, 2009) used a 3-level HMDP to evaluate the cost of clinical mastitis. A stage at level 0 models the life of a cow and its successors, a stage at level 1 models a lactation, and a stage at level 2 a models a month inside the lactation. Also, different models based on ordinary MDP have been suggested to solve the dairy cow replacement problem (see, for example, Rajala-Schultz et al., 2000; Vargas et al., 2001; and Groenendaal et al., 2004).

Kennedy and Stott (1993) incorporated a Bayesian reevaluation of yield potential based on previously recorded yields into their model. A similar approach has been used by Yalcin and Stott (2000) and Stott (1994) on both SCC and yield.

Even though many studies exist about the dairy cow replacement problem, the use of the models in modern herd management systems is limited. The use has often been limited to calculation of the economic impact of various factors (e.g., mastitis at the academic level or as a tool used by experts to aid the farmer). This may be because MDP models are often large and complicated and need advanced computer skills to use, or because user-friendly interfaces have not been developed. Moreover, parameters in the biological models used by the MDP must be estimated (i.e., data collection frameworks at herd-level must exist). Finally, in all of the studies mentioned above, the length of a stage in the MDP is from 1 mo up to $1 \mathrm{yr}$. Thus, the potential of these models is limited for assisting the farmer in everyday decision making such as when to inseminate, treat, or cull the cow in the current month.

In recent years, biosensors have been introduced in modern dairy herds and cow-specific traits and interventions are recorded in national databases. For example, automated milking systems can monitor the daily milk yield of a specific cow and the number of previous mastitis cases can be extracted from a database. As a result, a data collection framework exists that provides new information on a daily basis. However, dairy cow replacement models based on daily time steps are lacking.

The objective of this study was to formulate a dairy cow replacement model that can be integrated into a modern herd management system. Such a model should be based on daily milk recordings from biosensors, and parameters used in the models must be estimated using data records of the specific herd under consideration. The present paper describes the first step of developing such a model.

The study differs from previous contributions in that a multilevel HMDP was formulated using daily steps that can assist the farmer on a daily basis. Daily milk recordings from a biosensor in the milking system were used to predict the cow's performance dynamically (i.e., Bayesian updating was used to predict the performance of each cow in the herd and economic decisions are based on the prediction). Parameters used in the models were estimated using data records of the specific herd under consideration (including lactation curves).

\section{MATERIALS AND METHODS}

The dairy cow replacement problem was modeled using an HMDP (Kristensen and Jørgensen, 2000). In the model, a cow's life was represented as a sequence of stages. At each stage the cow was described using a set of states that identified different traits of the cow (e.g., milk production level and pregnancy). A cow could move from one state to another between stages (e.g., from being open in the current stage to becoming pregnant in the next). At a stage, the next state of the cow was not known with certainty. The transition probability to the next state depended on the current state and the decision made and could be found using biological models for the cow. We first introduce the biological models used and then describe the HMDP.

\section{Biological Functions}

Five biological models related to milk yield, reproduction, involuntary culling, growth, and feeding were used to estimate traits of the cow.

Milk Yield. The lactation curve or the average daily milk yield $\left(\mu_{t, j}\right)$ at herd level for each day $t$ and lactation $j$ was estimated using a mixed model and a spline function. Using a herd management system these curves could be estimated, for example, monthly using herd data from the last year. Other known curves for milk yield could also be used (Wood, 1967; Wilmink, 1987). However, in the context of herd decision support we were typically interested in lactation curves covering longer lactation periods than are used for breeding value estimations. Hence, spline curves were more flexible and thus more resistant to systematic bias in the last parts of the lactation period.

Because daily milk yield measurements were available and the individual cow may differ substantially from the herd mean (Figure 1a), a state space model (SSM) was formulated. Consider a cow in a dairy herd and let $M_{t, j}$ be the milk yield in kilograms of the cow at day $t$ (measured as days from calving) in lactation $j$. To keep the notation simple we did not consider indices for the cow and herd. We assumed the following model

$$
M_{t, j}=\mu_{t, j}+A_{j}+X_{t, j}+\nu_{t, j}
$$


where $\mu_{t, j}$ is the average herd milk yield on day $t$ at lactation $j$. Random variable $A_{j}$ denotes the production potential of the specific cow in lactation $j$. It is assumed that $A_{j} \sim N\left(0, \sigma_{A}^{2}\right)$. Random variable $X_{t, j}$ is an autoregressive process of order 1 with mean zero and autocorrelation $\rho(t)=\rho^{t},|\rho|<1$ (i.e., $X_{t, j}=\rho X_{t-1, j}+\varepsilon_{t, j}$ ). $X_{t, j}$ may be considered to be a local production effect on milk yield as a result of temporary environmental effects. Finally, $\nu_{t, j}$ are mutually independent random variables describing the measurement error. It was assumed that $\nu_{t, j} \sim N\left(0, \sigma_{\nu}^{2}\right)$.
If we subtracted the average daily yield on both sides of equation [1], we got the residual milk yield

$$
Y_{t, j}=M_{t, j}-\mu_{t, j}=A_{j}+X_{t, j}+\nu_{t, j}
$$

To illustrate, the daily milk yield $\left(M_{t, 3}\right)$ for 3 cows at lactation 3 in a Danish test herd is shown in Figure 1a. The average daily yield curve for the herd $\left(\mu_{t, 3}\right)$ is given by the solid black line. Note that cow 1 produced below average, cow 2 produced around average, and cow 3 produced above average. The corresponding residual
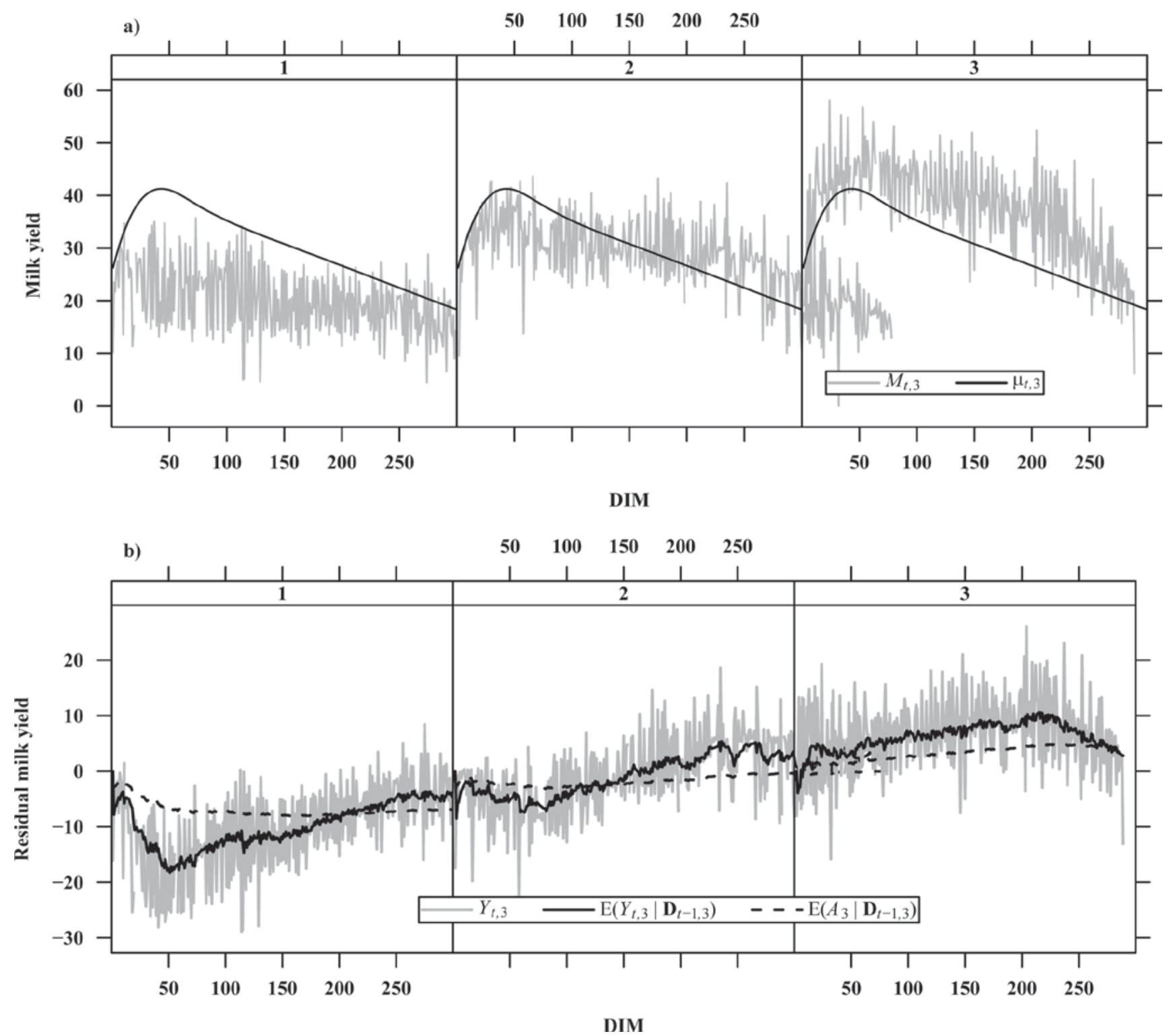

Figure 1. Milk yield and residual milk yield $(\mathrm{kg})$ for 3 different cows (lactation 3). (a) Daily milk yield $\left(M_{t, 3}\right)$ and estimated average herd yield curve $\left(\mu_{t, 3}\right)$. (b) Residual milk yield $\left(Y_{t, 3}\right)$, expected residual yield $\left[\mathbb{E}\left(Y_{t, 3} \mid \mathbf{D}_{t-1,3}\right)\right]$, and expected production potential $\left[\mathbb{E}\left(A_{3} \mid \mathbf{D}_{t-1,3}\right)\right]$. 
milk yield $\left(Y_{t, 3}\right)$ is shown in Figure 1b. A $Y_{t, 3}$ value above zero denotes that the yield was above average.

An SSM of equation [2] was specified for each lactation $j$ using the following measurement equation and system equation:

$$
\begin{gathered}
Y_{t, j}=\mathbf{F}^{\prime}\left(\begin{array}{c}
A_{j} \\
X_{t, j}
\end{array}\right)+\nu_{t, j}, \quad \mathbf{F}=\left(\begin{array}{l}
1 \\
1
\end{array}\right) \\
\left(\begin{array}{c}
A_{j} \\
X_{t, j}
\end{array}\right)=\mathbf{G}\left(\begin{array}{c}
A_{j} \\
X_{t-1, j}
\end{array}\right)+\mathbf{w}_{t, j}, \quad \mathbf{G}=\left(\begin{array}{ll}
1 & 0 \\
0 & \rho
\end{array}\right), \mathbf{w}_{t, j}=\left(\begin{array}{c}
0 \\
\varepsilon_{t, j}
\end{array}\right),
\end{gathered}
$$

where $\mathbf{w}_{t, j} \sim N(0, \mathbf{W})$, with

$$
\mathbf{W}=\left(\begin{array}{cc}
0 & 0 \\
0 & \left(1-\rho^{2}\right) \sigma_{X}^{2}
\end{array}\right) .
$$

Equation [3a] is equation [2] written using matrix notation, and equation [3b] describes the relationship between the latent variables from time $t-1$ to time $t$. W denotes the covariance matrix to random variable $\mathbf{w}_{t, j}$.

We assume that the prior is normal distributed, $\left(A_{j}, X_{0, j}\right)^{\prime} \sim N\left(\mathbf{m}_{0, j}, \mathbf{C}_{0, j}\right)$, with mean and covariance matrix

$$
\mathbf{m}_{0, j}=\left(\begin{array}{c}
\hat{A}_{0, j} \\
0
\end{array}\right), \quad \mathbf{C}_{0, j}=\left(\begin{array}{cc}
\sigma_{A}^{2} & 0 \\
0 & \sigma_{X}^{2}
\end{array}\right),
$$

where $\hat{A}_{0, j}$ denotes our expectation to the production potential of the cow in lactation $j$ before no daily milk yield records are available. We let $\hat{A}_{0,1}=0$ (i.e., we expected the cow to be an average heifer when it was inserted in the herd). For $j>1$ we set $\hat{A}_{0, j}$ equal to the expected production potential at the end of lactation $j-1$ (i.e., we expected the production potential to be the same as estimated during the last lactation). Note that an expected production potential of 10 at the end of lactation 1 meant that the cow was estimated to produce $10 \mathrm{~kg}$ of milk above the average herd milk yield curve of lactation 1 . If the expected production potential for a cow in the start of lactation 2 was set equal to 10 , the cow was expected to produce $10 \mathrm{~kg}$ of milk above the average herd milk yield curve of lactation 2 each day.

Let $\mathbf{D}_{t, j}=\left(Y_{t, j}, \ldots, Y_{t, j}, \mathbf{m}_{0, j}, \mathbf{C}_{0, j}\right)$ denote the daily milk yield records available up to time $t$ including prior information. Moreover, let $\mathbf{m}_{t, j}$ denote the conditional latent mean at time $t$ ( $\mathbb{E}$ denotes expectation):

$$
\mathbf{m}_{t, j}=\left(\begin{array}{c}
\hat{A}_{t, j} \\
\hat{X}_{t, j}
\end{array}\right)=\mathbb{E}\left(\left(\begin{array}{c}
A_{j} \\
X_{t, j}
\end{array}\right) \mid \mathbf{D}_{t, j}\right) .
$$

Given the posterior of the latent variable at time $t-1\left[\left(A_{j}, X_{t-1, j}\right)^{\prime} \mid \mathbf{D}_{t-1, j}\right] \sim N\left(\mathbf{m}_{t-1, j}, \mathbf{C}_{t-1, j}\right)$, we used the Kalman filter to predict the residual daily milk yield at time $t$ (West and Harrison, 1997, Theorem 4.1):

$$
\mathbb{E}\left(Y_{t, j} \mid \mathbf{D}_{t-1, j}\right)=\hat{A}_{t-1, j}+\rho \hat{X}_{t-1, j}
$$

The expected daily milk yield at time $t$ can be found by adding the average herd yield:

$$
\mathbb{E}\left(M_{t, j} \mid \mathbf{D}_{t-1, j}\right)=\mathbb{E}\left(Y_{t, j} \mid \mathbf{D}_{t-1, j}\right)+\mu_{t, j} .
$$

The above results show how to predict the residual daily milk yield based on data records available. For example, the expected residual milk yield $\left[\mathbb{E}\left(Y_{t, 3} \mid \mathbf{D}_{t-1,3}\right)\right]$ of the 3 cows in Figure 1 is shown with a solid black line in Figure 1b. Similarly, if we set the prior estimate of $A_{3}$ at $t=0$ to zero, the expected value of $A_{3}$ slowly adapted to the production level of the cow. A value above zero corresponded to a cow predicted to yield better than an average cow and a value below zero to a cow predicted to yield worse than average. The expected value of $A_{3}$ was important because in a decision model a high expectation will correspond to a highyielding cow that we want to keep longer than a lowyielding cow. A description of the estimation of the lactation curves and the variance parameters in the SSM is given in the appendix, which also contains a plot of $\mu_{t, j}$.

Growth. The total BW of the cow was based on a combination of 2 curves: 1) a Gompertz curve used to describe the standardized $\mathrm{BW}$ of the animal corrected to a body BCS of 3 (5-point scale) and excluding any weight of a fetus (Kristensen et al., 2008), and 2) a BCS curve yielding the BCS during the lactation based on Friggens et al. (2004), which assumed that the BCS at the start of the lactation decreases until a genetically determined time in the lactation, stays the same until conception, and afterwards increases until the end of the lactation. A mathematical description of the growth functions and an overview of the parameters are given in the appendix.

Feeding and Energy. The daily feed intake of the cow was measured in net energy of Scandinavian feed units (SFU). The total amount of energy was the sum 


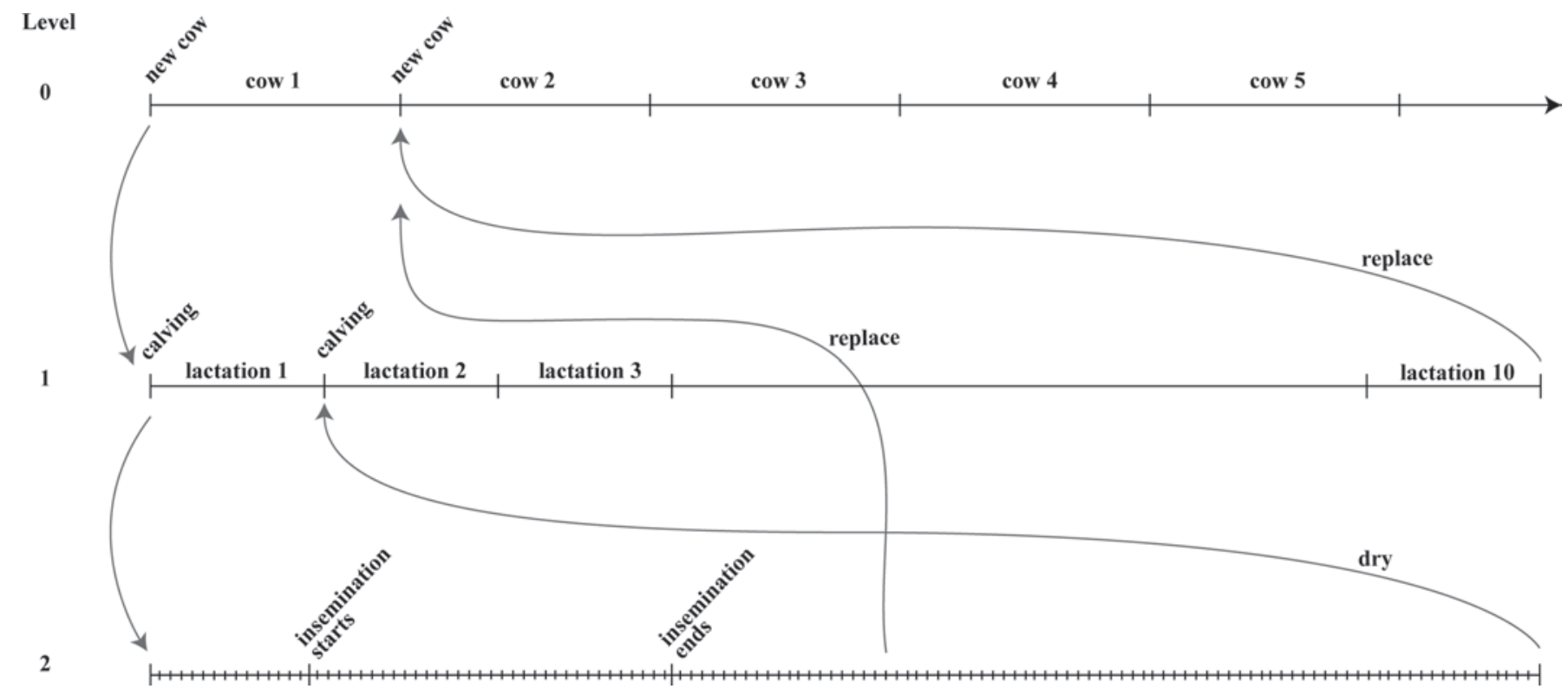

Figure 2. The different levels in the hierarchical Markov decision process. Each time instance corresponds to a set of states.

of energy needed for maintenance, fetus growth, milk yield, BCS-corrected BW gain, and BCS change. An average daily intake of 7 SFU was assumed over a dry period of $49 \mathrm{~d}$. All functions were taken from Kristensen et al. (2008) and Østergaard et al. (2005). For an explicit description of the energy functions and an overview of the parameters, see the appendix.

Reproduction. A model for reproduction that simulates the estrus cycle was constructed. It modeled the waiting time from calving until the farmer knew that the cow was pregnant and was based on a continuous Markov chain describing the estrus cycle, and included a voluntary waiting period (insemination was not started before $\mathrm{d}$ 35). Moreover, $40 \mathrm{~d}$ after an insemination a pregnancy test was taken. The probability of a positive pregnancy test could be found using the model. Finally, if the cow had not become pregnant at d 250, insemination was discontinued. A description of the reproduction model is given in the appendix, which also contains a plot of the probability of a positive pregnancy test.

Involuntary Culling. Disposal of cows that was not subject to decision making in the model was referred to as involuntary culling (IC). Reasons for IC could be dead cows and cows sold for slaughter because of reasons other than milk yield and failure to conceive (e.g., illness). Involuntary culling would typically be influenced by factors such as lactation stage, disease incidence, and age of the cow. We formulated a model that could capture the major feature of this pattern. The conditional daily probability for IC was based on a piecewise linear function for the hazard rate. The hazard rates were based on previous literature (e.g., Beaudeau et al., 1995; Dechow and Goodling, 2008) but modified such that the probabilities varied within each lactation depending on, for example, pregnancy status instead of only days from calving. For a detailed description of the probability of involuntary culling and a plot, see the appendix.

\section{HMDP Formulation}

The method used for optimization was based on an HMDP with multiple levels. For a detailed description of the method, see Kristensen and Jørgensen (2000).

Model Structure. The model was constructed as a 3-level HMDP as illustrated in Figure 2, which gives a graphical overview over the process. At level 0 only a single process (the founder process) existed with an infinite time-horizon. Each stage represented the lifetime of a cow (i.e., the process represented the current cow and all its successors). For each stage of the founder process (i.e., the life of a cow) the duration and reward of the stage were defined by a child process at level 1 , which was a finite time-horizon MDP with 10 stages. Each stage corresponded to a possible lactation, which again was expanded to a child process at level 2. Here the lactation was divided into daily stages. At level 2 the process ended when the cow was dried off and the process returned to the next stage (lactation) at level 1 or if the cow was replaced, which resulted in the process returning to the founder level (a new cow was inserted). 
Stage Length, States, and Decisions. At level 0 only a dummy state was defined, representing that a new cow was inserted. At level 1 a state was defined as the cow's expected production potential (13 levels; i.e., $\hat{A}_{t, j}$ of equation [5]). The stage length at levels 0 and 1 was defined by its child processes. In the model, at most 10 stages were allowed at level 1 ; that is, the maximum age of the cow was 10 lactations, which was similar to other MDP models for dairy cows (Houben et al., 1994; Vargas et al., 2001).

A state at level 2 was defined as a combination of the following state variables: the pair $(\hat{A}, \hat{X})$, that is, the expected production potential and the expected local production effect (a set $\Phi_{(\hat{A}, \hat{X})}$ containing 45 levels), and drying off week $\hat{w}$ (a set $\Phi_{\hat{w}}$ containing 32 levels), which was a measure for the calving interval. The choice of drying off week instead of drying off day was the result of a need to keep the number of states in the HMDP small. Moreover, a state was added to represent that the cow had been involuntary culled.

The set $\Phi_{(\hat{A}, \hat{X})}$ was a discrete approximation of the 2-dimensional space of the expected production potential and the expected local production effect. The approximation was made using the nonuniform discretization (for more details, see the appendix).

Because the possible period for insemination was from d 35 to d 250 and the dry period according to Danish standards was set to $7 \mathrm{wk}$ before calving, the possible weeks to dry the cow were $\Phi_{\hat{w}}=\{39,40$, $41, \ldots, 69,-1\}$. The value -1 indicated that the drying off week was not yet determined. This was the case if we did not have a positive pregnancy test (taken $40 \mathrm{~d}$ after insemination). Not all states were possible at each stage (e.g., the drying off week was unknown before $d$ 75 and hence only states with drying off week equal to -1 were possible).

We dried the cow at the end of the drying off week. Furthermore, 2 decisions were possible at level 2. Either we could choose to keep the cow or we could replace it. In the first case the process continued to the next stage and in the second the process returned to level 0 (a new cow was inserted). Because the cow was replaced immediately, the length of the stage was zero. Because of public regulations it was not possible to replace the cow in the last tenth of the pregnancy.

The stage length between 2 stages at level 2 was $1 \mathrm{~d}$, except the last stage corresponding to the dry period, which had a length of $49 \mathrm{~d}$. Because the maximum legal week to dry the cow was wk 69 , the maximum length of a lactation in the model was $532 \mathrm{~d}(69 \times 7+49 \mathrm{~d})$. Moreover, we assumed that if the cow did not become pregnant it may be kept at most until d $483(69 \times 7 \mathrm{~d})$, at which time it was replaced.

Transition Probabilities. Consider a child process at level 2 representing lactation $j$ and let

$$
s_{t} \in\left\{(\hat{A}, \hat{X}, \hat{w}) \mid(\hat{A}, \hat{X}) \in \Phi_{(\hat{A}, \hat{X})}, \hat{w} \in \Phi_{\hat{w}}\right\} \cup\{\mathrm{IC}\}
$$

denote a state at stage $t$. That is, $s_{t}$ was either a triple $(\hat{A}, \hat{X}, \hat{w})_{t}$ describing the yield and the week of drying the cow or the IC state. Let week $(t)$ denote a function that calculates the week number given day $t$. If a positive pregnancy test was detected at day $t$, then the drying off day was $t$ - pregnancy test delay + pregnancy length - dry period length $=t+193$; that is, the drying off week was week $(t+193)$.

Given state $(\hat{A}, \hat{X}, \hat{w})_{t-1}$ with $\hat{w}_{t-1}=-1$ (pregnancy status unknown) and decision keep, we had that

$$
\begin{aligned}
& p\left[s_{t} \mid(\hat{A}, \hat{X}, \hat{w})_{t-1}, j\right] \\
& = \begin{cases}p_{I C} & s_{t}=\mathrm{IC} \\
\left(1-p_{I C}\right) \times p_{P T} \times p_{(\hat{A}, \hat{X})} & \hat{w}_{t}=\operatorname{week}(t+193) \\
\left(1-p_{I C}\right) \times\left(1-p_{P T}\right) \times p_{(\hat{A}, \hat{X})} & \hat{w}_{t}=-1 \\
0 & \text { otherwise }\end{cases}
\end{aligned}
$$

where $p_{I C}$ denotes the probability of involuntary culling (see the appendix on functions related to culling) and $p_{P T}=\operatorname{prPosPregT}(t)$ denotes the probability of a positive pregnancy test (see the appendix on functions related to reproduction). The probability $p_{(\hat{A}, \hat{X})}=\operatorname{prM}\left[(\hat{A}, \hat{X})_{t},(\hat{A}, \hat{X})_{t-1}, t\right]$ denotes the probability of a transition from $(\hat{A}, \hat{X})_{t-1}$ to $(\hat{A}, \hat{X})_{t}$; see the appendix on discretizing the SSM. Under decision keep with a known pregnancy status (i.e., $\hat{w}_{t-1}>0$ ), we had

$$
\begin{aligned}
& p\left[s_{t} \mid(\hat{A}, \hat{X}, \hat{w})_{t-1}, j\right] \\
& = \begin{cases}p_{I C} & s_{t}=\mathrm{IC} \\
\left(1-p_{I C}\right) \times p_{(\hat{A}, \hat{X})} & \hat{w}_{t}=\hat{w}_{t-1} \\
0 & \text { otherwise }\end{cases}
\end{aligned}
$$

That is, the week of drying off the cow was fixed when the pregnancy status became known. 
If we chose to replace the cow, the child process terminated and returned to the founder process with probability 1 . At the last day in wk $\hat{w}_{t}$, we dried off the cow. This resulted in the child process terminating and returning to the next stage of its parent process (next lactation). Finally, if the cow entered the IC state, it was replaced and the process returned to the founder process with probability 1 .

Rewards and Milk Yield. The rewards of the model were equal to the expected economic net revenue of a cow during the stage in question given all information available (i.e., the stage, state, and action of the process and ancestral information). At levels 0 and 1 , all rewards and milk yields were calculated based on their child process. The cost in the initial state of level 1 (which had no child process) was the cost of buying the heifer.

At level 2 the reward under decision replace was calculated as the carcass weight multiplied by a fixed carcass price. The carcass weight was set to fixed proportion of the live weight of the cow (Vestergaard et al., 2007).

The reward under decision keep was calculated as revenues from milk minus feeding costs multiplied by the survival rate ( 1 minus the probability of IC) plus the reward of replacing the cow (calculated as under decision replace) multiplied by the probability of IC. The revenues from milk were given as the milk yield in ECM multiplied by a fixed milk price. The feeding costs were given as the energy need in SFU multiplied by a fixed SFU price.

The reward under decision dry was calculated as revenues from the calf minus feeding costs multiplied by the survival rate (1 minus the probability of IC) plus the reward of the carcass multiplied by the probability of IC. The feeding cost was given as the energy needed during the dry period times the SFU price. Only the decision to keep the cow generated a positive milk yield, which could be calculated using equation [7] and converted to ECM assuming a fixed level of fat and protein (fat $4.12 \%$ and protein $3.38 \%$ ).

Optimizing the HMDP. A policy or strategy specifies the decisions to be used at all stages and states and provides the farmer with a plan of which decision to take given stage and state. Given the HMDP our objective was to find a policy that maximized the expected discounted net present value (NPV) using a specific discount rate. Because the NPV at a specific stage and state depended on the expected reward generated as a result of decisions taken at future states, the number of suboptimal policies was extremely large. However, total enumeration of the policies could be avoided by using a combination of value and policy iteration to find the policy that maximized the NPV. These are well-known optimization techniques and we refer the reader to Kristensen and Jørgensen (2000) for further details.

Parameters and Prices. The HMDP model could be optimized under various parameters related to the biological models and prices and hence was not restricted to specific parameters values. An overview of the parameters used in the biological models is given in the appendix. Parameters were estimated based on milk recordings from the robot milking system at a Danish dairy farm.

Prices used when optimizing the HMDP were taken from Danish market conditions mid-2008 and converted from Danish kroner (DKK) to Euro (€) using an exchange rate of 7.46. The price of a heifer was $€ 1,153$. The carcass price was $€ 1.94 / \mathrm{kg}$. The milk price was $€ 0.38 / \mathrm{kg}$ of ECM. The price of an SFU was $€ 0.18$. The price of a calf was $€ 121$.

The HMDP was optimized with the program named MLHMP (Kristensen, 2003), maximizing the expected discounted NPV. A yearly interest rate of $2.5 \%$ was used. The total number of states in the model was $3,011,884$. This included only the states of 1 child process at level 2 . Note that a child process at level 2 was defined for each of the 13 states at level 1 . However, all these child processes were equal except for their initial transition probabilities, which were dependent on the state at level 1 (the cow's expected production potential). As a result, only a single child process at level 2 and the initial distribution had to be stored. This reduced the number of states in the model that otherwise would have been approximately 13 times higher.

\section{RESULTS}

Given a specific cow, lactation and time instance (DIM), the expected production potential $\left(\hat{A}_{t, j}\right)$ and the expected local production effect $\left(\hat{X}_{t, j}\right)$ could be estimated using the SSM for the yield [i.e., the value of $\left(\hat{A}_{t, j}, \hat{X}_{t, j}\right)$ would be updated during the lactation based on the daily yield measurements].

The trajectory of $\left(\hat{A}_{t, j}, \hat{X}_{t, j}\right)$ are illustrated in Figure 3 for the 3 cows presented in Figure 1. Each point corresponds to a value of $\left(\hat{A}_{t, j}, \hat{X}_{t, j}\right)$ at a specific point in time. The number near a point is DIM. It can be seen that the expected production potential for cow 1 was below zero during the lactation, for cow 2 around zero, and for cow 3 above zero, indicating whether the cows were producing below or above an average cow in the herd. The expected local production effect fluctuated more because this effect was more sensitive.

At a specific time instance, the value $\left(\hat{A}_{t, j}, \hat{X}_{t, j}\right)$ together with lactation number and pregnancy status 


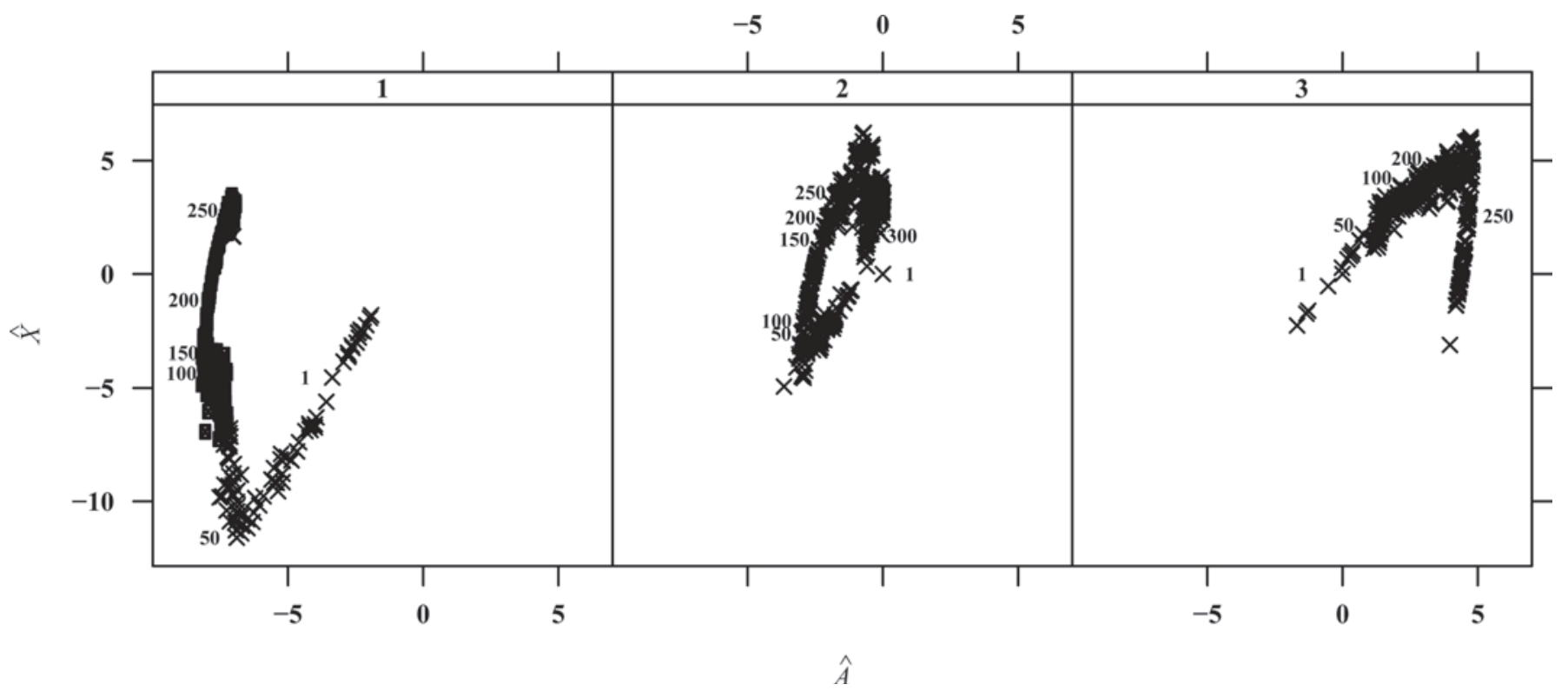

Figure 3. The trajectory of the expected production potential $(\hat{A})$ and the expected local production effect $(\hat{X})$ for the cows in Figure 1 (lactation 3). Each point corresponds to a value of $(\hat{A}, \hat{X})$ at a specific point in time. The shape of the point indicates whether the optimal decision is to keep or replace (cross $=$ keep; dot $=$ replace). The number near a point is DIM.

could be used to identify the state of the HMDP, and the optimal policy calculated specified which decision to take. The optimal decision is shown in Figure 3 using the shape of the point, which indicates whether the optimal decision is to keep or replace (dot $=$ keep; cross = replace).

Opportunity costs could also be calculated. Let the retention payoff (RPO) denote the difference between the NPV under decision keep and NPV under decision replace for a specific state in the HMDP (i.e., if $\mathrm{RPO}$ was positive the optimal decision was to keep the cow and if RPO was negative the optimal decision was to replace the cow). The RPO is the total extra (discounted) reward of keeping the cow until her optimal replacement time.

Figure 4 shows the RPO for the 3 cows presented in Figure 1. The horizontal line in the bottom of each plot indicates whether the optimal decision is to keep or replace (gray $=$ keep; black $=$ replace). Vertical lines correspond to start of lactation (s), inseminated (i), positive pregnancy test (p), and dry (d). Cow 1 was optimal to replace $45 \mathrm{~d}$ from calving whereas cows 2 and 3 were recommended kept under the whole lactation. Note that RPO would never be very negative because a negative RPO described the loss of keeping the cow 1 more day.

Although the value of $\left(\hat{A}_{t, j}, \hat{X}_{t, j}\right)$ would change during lactation for a specific cow, it was of interest how NPV and RPO would develop during lactation for fixed
$\left(\hat{A}_{t, j}, \hat{X}_{t, j}\right)$.The NPV over 10 lactations for a cow assumed dried off in wk 54 is shown in Figure 5. Each line corresponds to different values of $\left(\hat{A}_{t, j}, \hat{X}_{t, j}\right)$. The expected local production effect $\left(\hat{X}_{t, j}\right)$ is assumed zero in the figure. Figure 5 illustrates the importance of the production potential of the cow. Changes in expected production potential that resulted in changes in the expected daily yield less than $10 \mathrm{~kg}$ had a high effect on NPV. If we compare lactations, a cow in her second lactation had the highest reward that afterwards decreased with lactation number. Inside a lactation, the NPV of the cow changed. In general, the NPV was first high in lactation and then decreased until pregnancy was discovered, at which point the NPV may jump to a higher value. Afterwards NPV may decrease for a period and subsequently increase until the end of the lactation. One exception was for a cow in her 10th lactation. Here the NPV only decreased.

The RPO for lactations 1 to 3 is shown in Figure 6 for a cow assumed dried off in wk 54. Each line corresponds to different values of $\left(\hat{A}_{t, j}, \hat{X}_{t, j}\right)$. The RPO followed a pattern almost equal to NPV. It can be seen that cows with the same expected production potential but a different local production effect had different $\mathrm{RPO}$ at the start of the lactation but equal RPO at the end of each lactation. The RPO of cows with the same expected daily yield may be quite different (see lines with expected yield equal to 7.67 and 7.54 ). Only cows 


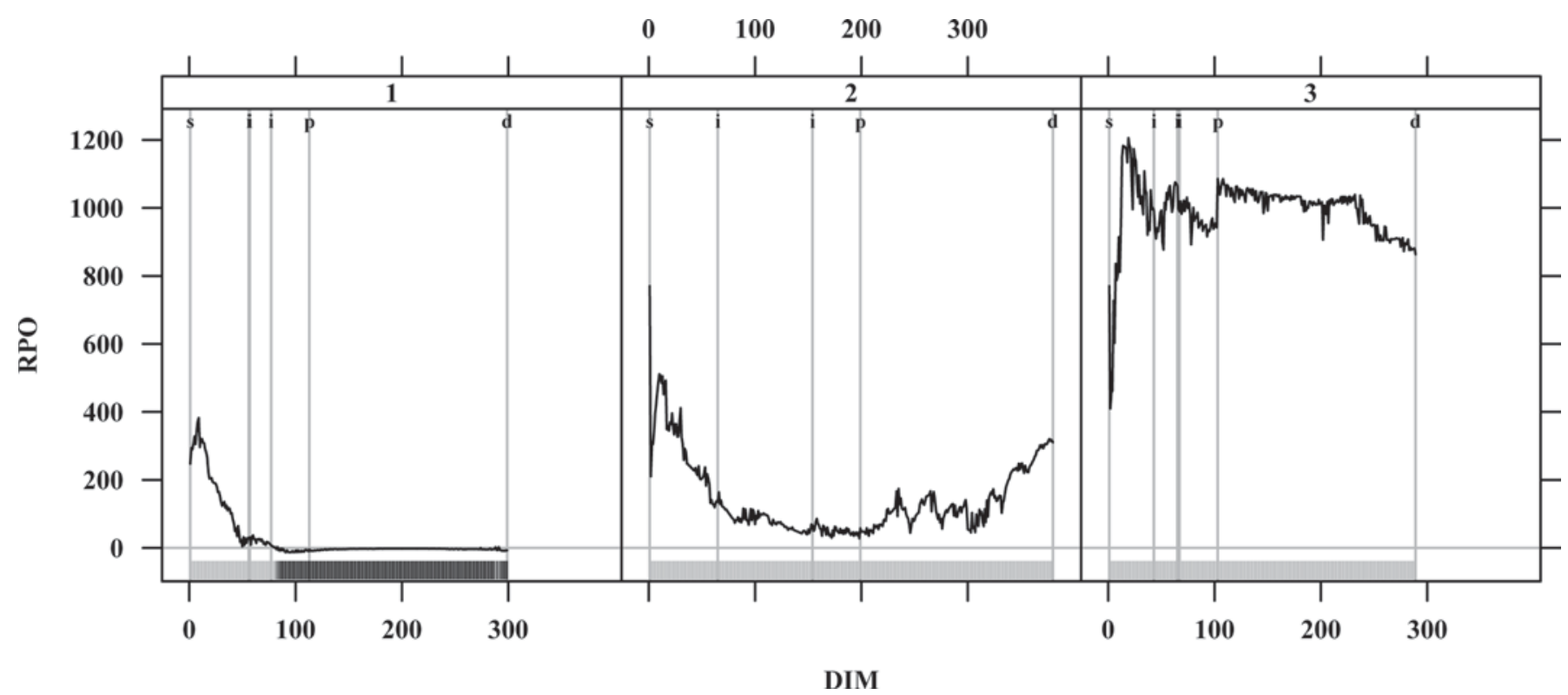

Figure 4. Retention payoff (RPO) in Euros for the cows in Figure 1 (lactation 3). The horizontal line in the bottom of each plot indicates whether the optimal decision is to keep or replace (gray $=$ keep; black = replace). Vertical lines correspond to start of lactation $(\mathrm{s})$, inseminated (i), positive pregnancy test (p), and dry (d).

with low production potential were recommended replaced during lactation. Moreover, if the cow because of other circumstances had not been replaced, it may have been optimal to keep her for another lactation because
RPO increased and became positive again at the end of the lactation.

Changes in the parameters of the model also affected the model output as shown in Table 1. Here, 4 scenarios

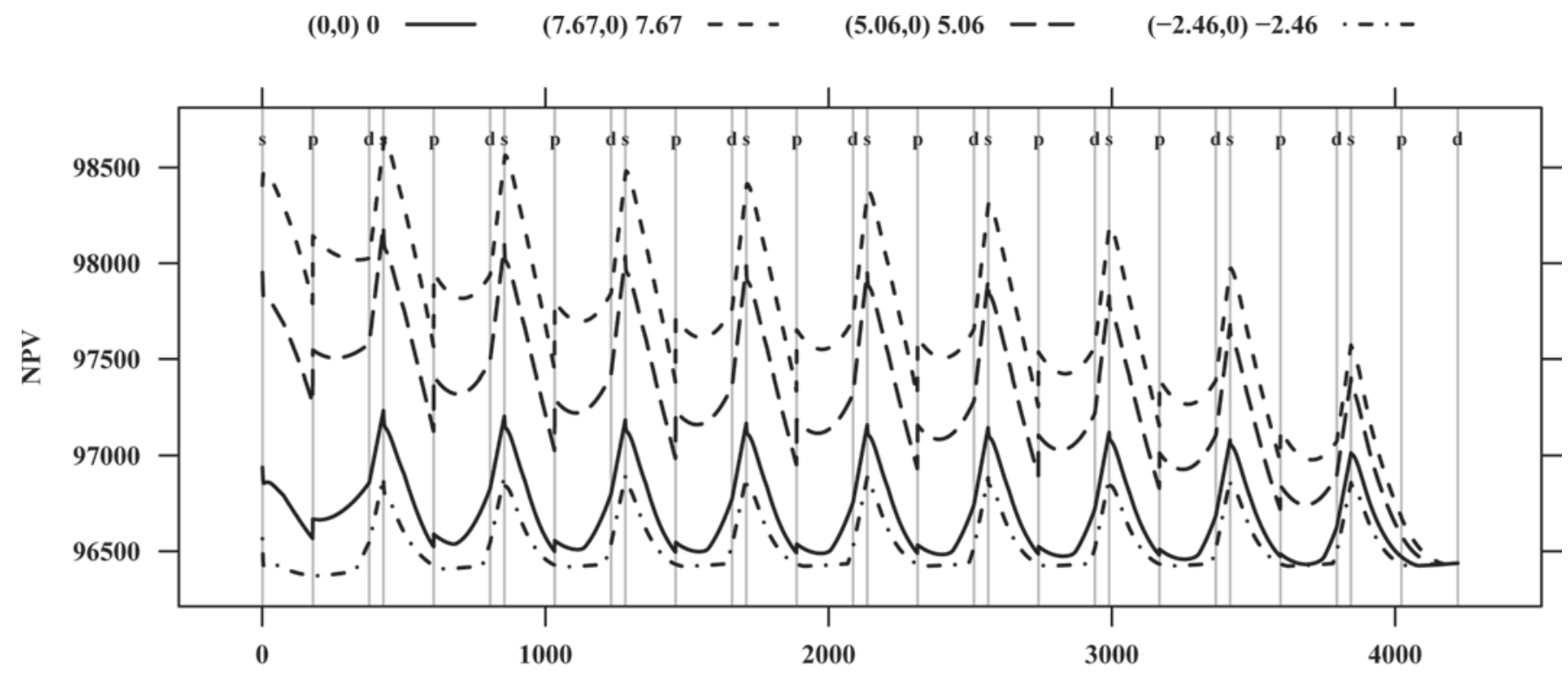

Days from start of lactation one

Figure 5. Net present value (NPV) in Euros over 10 lactations for a cow assumed dried off in wk 54. Each line corresponds to a different value of the expected production potential $\left(\hat{A}_{t, j}\right)$ assuming that the expected local production effect $\left(\hat{X}_{t, j}\right)$ is zero. The number reported in the legend is first $\left(\hat{A}_{t, j}, \hat{X}_{t, j}\right)$ and second the expected daily yield (ECM). Vertical lines correspond to start of lactation (s), positive pregnancy test (p), and dry (d). 
Table 1. The effects of multiplying the probabilities of involuntary culling (IC) by 1.25 and 0.75 , increasing the heat detection probability by $30 \%$, and delaying the start of the insemination period by $20 \mathrm{~d}$ compared with the basic scenario

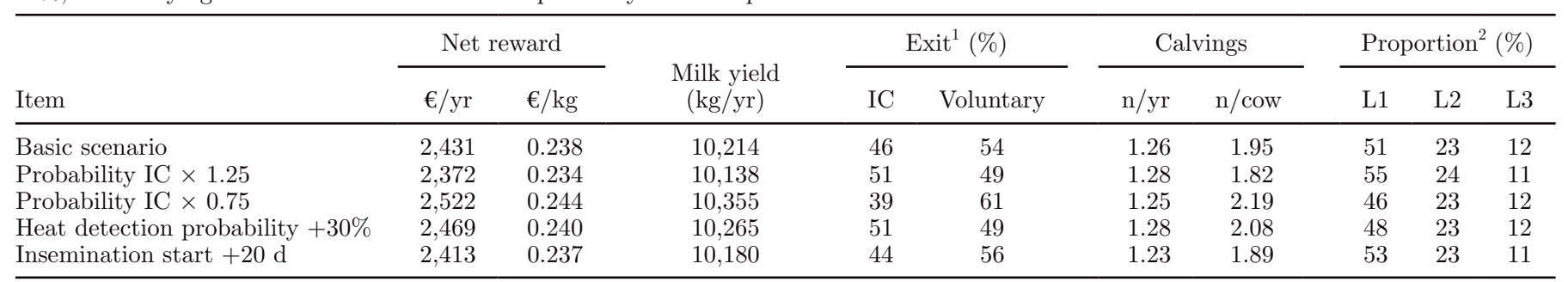

${ }^{1}$ Percentage culled.

${ }^{2}$ Proportion of total calvings over lactations (L) 1 to 3 in the herd.

are compared with the basic scenario. In the first 2 scenarios we increased and decreased the probability of IC by $25 \%$ (i.e., the parameters of the IC model described in the appendix were multiplied by 1.25 and 0.75 , respectively). In the next scenario the probability of heat detection during the lactation was increased by $30 \%$. Finally, in the last scenario the day at which insemination started was increased $20 \mathrm{~d}$, from d 35 to d 55.

An increase in IC, as expected, reduced the net reward and decreased the number of calvings per cow, which increased the proportion of calvings in the first lactation. A decrease in IC had the opposite effect. A better heat detection generated more calvings for each cow during her reproductive life, resulting in a lower proportion of first lactation calvings. Finally, delaying the start of insemination by $20 \mathrm{~d}$ cut net rewards and decrease calvings.

\section{DISCUSSION}

The model presented in this paper is a step in the direction of developing an HMDP model that can be integrated into a modern herd management system. An

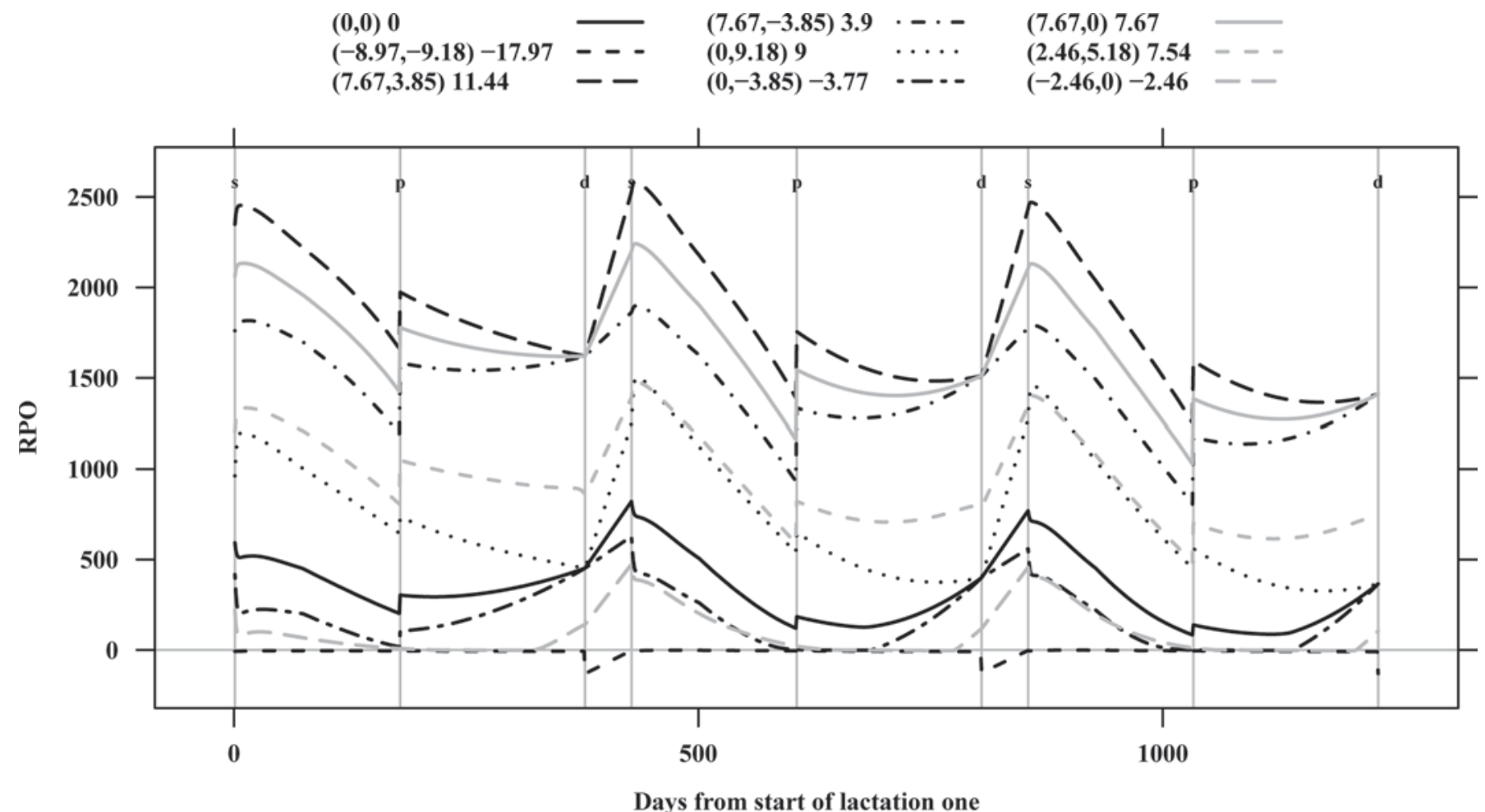

Figure 6. Retention payoff (RPO) in Euros over lactations 1 to 3 for a cow dried off in wk 54 . Each line corresponds to a different expected production potential $\left(\hat{A}_{t, j}\right)$ and expected local production effect $\left(\hat{X}_{t, j}\right)$. The number reported in the legend is first $\left(\hat{A}_{t, j}, \hat{X}_{t, j}\right)$ and second the expected daily yield (kilograms). Vertical lines correspond to start of lactation (s), positive pregnancy test (p), and dry (d). 


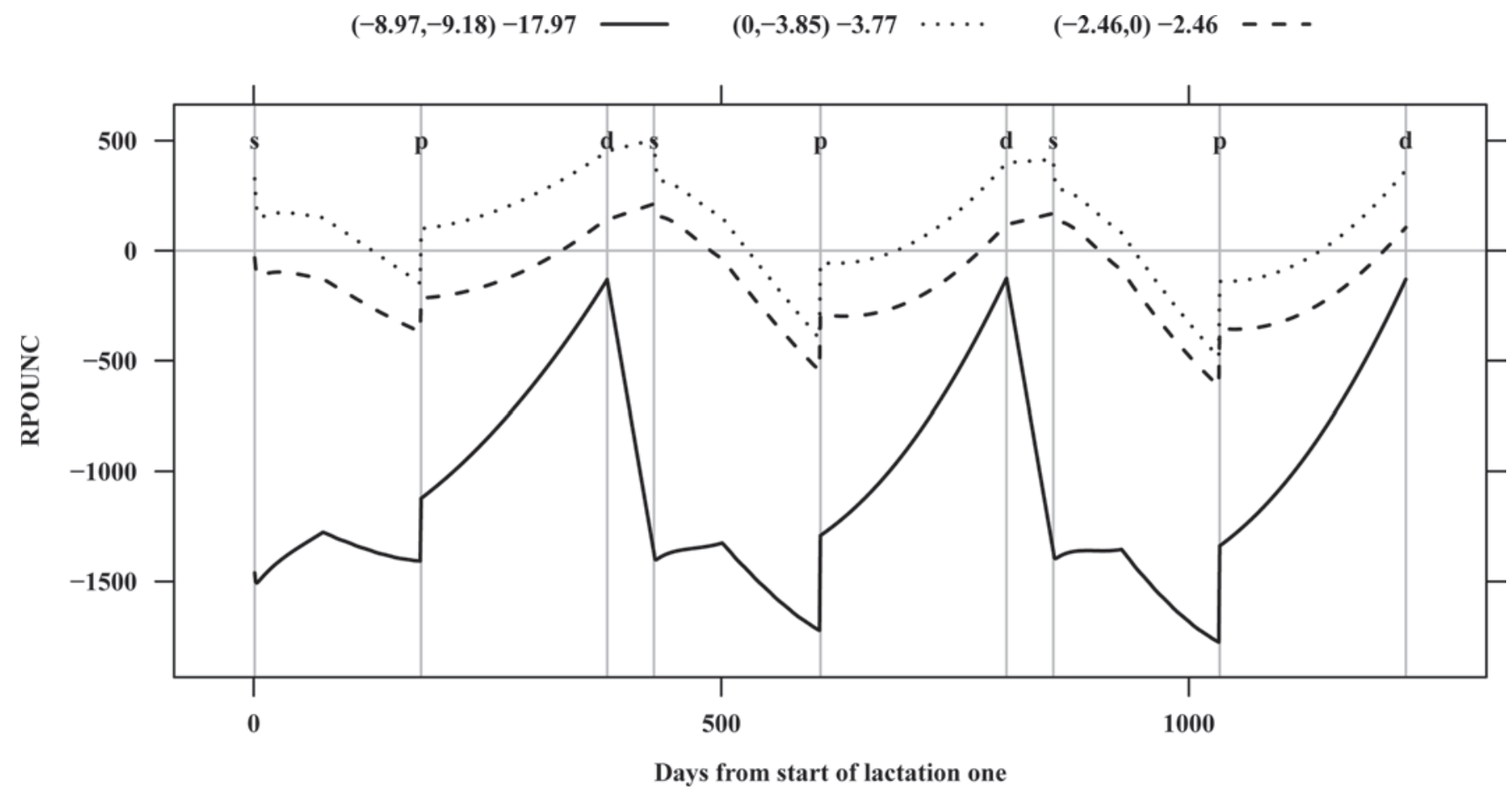

Figure 7. Retention payoff until next calving (RPOUNC) in Euros over lactations 1 to 3 for a cow dried off in wk 54. Each line corresponds to a different expected production potential $\left(\hat{A}_{t, j}\right)$ and expected local production effect $\left(\hat{X}_{t, j}\right)$. The number reported in the legend is first $\left(\hat{A}_{t, j}, \hat{X}_{t, j}\right)$ and second the expected daily yield (kilograms). Vertical lines correspond to start of lactation (s), positive pregnancy test (p), and dry (d).

SSM was formulated that, based on daily milk records from the milking system, can predict the cow's production potential and local production effect (Figure 3). Using this model together with a Kalman filter, we did not have to store all previous yield records because the expected production potential and local production effect could be found using the last yield record and the prior distribution of the production potential and local production effect. The parameters in the SSM were estimated using data records of the specific herd under consideration. This included herd-specific lactation curves.

Economic optimal values and decisions were calculated using the HMDP model, which calculates optimal decisions that assist the farmer on a daily basis in deciding when to replace the cow. Moreover, economic values such as NPV and RPO for each cow can be provided to the farmer on a daily basis. The HMDP is novel compared with earlier MDP models such as Houben et al. (1994) and Bar et al. (2008) because we considered time steps of $1 \mathrm{~d}$ and an SSM model for the yield. The SSM was embedded into the HMDP such that economic decisions were based on the prediction of the performance of the cow under consideration. Note that the daily milk yield records were not represented in the HMDP model. Only the latent variables $\left(\hat{A}_{t, j}, \hat{X}_{t, j}\right)$ of the SSM have to be represented as state variables in the HMDP. This reduced the number of states in the model.

The NPV illustrated in Figure 5 for different expected production potentials shows the importance of the production potential of the cow. Changes in the expected daily yield (less than $10 \mathrm{~L}$ ) had a high effect on NPV. The NPV was highest in the second lactation and afterwards it decreased with lactation number. This was also the result found in Heikkila et al. (2008).

During the lactation the NPV varied. It was high in the beginning of the lactation and decreased until pregnancy. This was a result of the form of the milk yield lactation curve with high yield in the start of lactation. Next, when pregnancy was discovered the NPV may jump to a higher value. This was because when a cow was discovered pregnant, there was a much higher probability of keeping it for an additional lactation. Finally, after pregnancy was discovered the NPV may decrease for a period and then it may increase until the end of the lactation. This was a result of $2 \mathrm{ef}-$ fects: the lactation curve with decreasing milk yield and 
the expectation of high yield in the next lactation. At the beginning the first effect may dominate and after a period of time the second effect dominate.

The RPO was the total extra reward for keeping the cow until her optimal replacement time (i.e., if RPO was positive the optimal decision was to keep the cow and if RPO was negative the optimal decision was to replace the cow). From the RPO illustrated in Figure 6 it can be seen how the local production effect affects the RPO. For cows with the same expected production potential but different expected local production effect, the RPO varied much in the start of the lactation; however, the difference became smaller during the lactation. This was because the local production effect was not carried over between lactations (i.e., all cows were assumed to start with a local production effect equal to zero at the start of a lactation). This was a realistic assumption because the dry period is a complicated stage in the cow's life and the local effect was reset during this stage.

From Figure 6 it can also be seen that local production effect had a lower effect on the RPO compared with the production potential. If we compared cows with the same expected daily yield (see lines with expected yield equal to 7.67 and 7.54; Figure 6), the RPO would be highest if we have a high expected production potential.

Only cows with low expected production potential were recommended to be replaced during lactation, which was consistent with previous studies (Houben et al., 1994; Bar et al., 2008). If a cow because of some circumstance had not been replaced at her optimal time in the middle of lactation, then it may be optimal to keep her until the middle of the next lactation because RPO may increase and become positive again at the end of the lactation, as can be seen in Figure 6 for the lines with expected production potential and local production effect equal to $(0,-3.85)$ and $(-2.46,0)$.

Because a negative RPO was the loss of keeping the cow 1 more day, only small negative RPO values were possible. Therefore, negative RPO values may not be appropriate for illustrating the possible loss of keeping the cow. The farmer might argue to keep the cow for another day because the loss is very small. However, over some weeks the loss accumulates. A way to demonstrate this is to introduce a new economic measure that calculates the economic gain or loss over a longer period. One possibility is to define the retention payoff until next calving (RPOUNC), which is the difference between the NPV of keeping the cow until next calving and the NPV of replacing the cow now.
The RPOUNC is shown in Figure 7 for the 3 lines with the lowest RPO in Figure 6. Note that a negative RPOUNC is not equivalent with the decision to replace the cow, as in the case of RPO. Instead, RPOUNC provides the farmer with the loss or gain of keeping the cow until the next lactation.

In general, MDP models are difficult to validate because observed output data of the model is not available. The different submodels (e.g., the models of the lactation curves) can be validated, especially when the model selection and parameter estimation are based on the herd-specific recordings. In this case the standard statistical framework for model check and model comparison were available to ensure sufficient validity of the submodel. The analysis summarized in the appendix includes these steps.

With respect to the total model, approaches such as face validity have been suggested (Sørensen, 1990; Sargent, 2008); that is, the assessment of the validity of the model is based on techniques such as plots of results, input data validation, sensitivity analysis, and comparison to other models. These techniques are similar to techniques used for model verification and as such are a natural part of the model-building process.

A more strict validation of the model in our case would correspond to designing a study in which the model was implemented on a set of farms and used by the farmers and then compared against a set of farms without access to the model. The output data from these 2 groups could then be used for model comparison and validity check. Such resources to analyze complex herd systems will seldom be available, as pointed out in the general discussion of a symposium concerning modeling of livestock production, where it was summarized that such models would be too expensive to test (Korver and van Arendonk, 1988; Verstegen et al., 1995).

The model presented in this paper is a simplification of the real world, as is any other model. The simplifications in different models can also be used to assess the validity of the model. The model is targeted to use daily yield measurements and thereby has daily time steps that allow exit from the lactation on a daily basis. This is an improvement compared with earlier MDP models that consider time steps of a month or year and do not have such a detailed description of the milk yield (Bar et al., 2008; Heikkila et al., 2008).

As pointed out above in the discussion section, the curves in the plots of NPV, RPO, and RPOUNC can be explained and seems reasonable. Furthermore, the sensitivity analysis conducted provides results as expected, which supports the validity of the model. 
The underlying biological functions used by the MDP are all based on statistical models. The milk yield model used Bayesian updating and predicted yield on a daily basis during lactation. Moreover, parameters of the model were based on herd data and not population data. That is, the prediction was more precise to the herd under consideration, which is an improvement compared with previous dairy replacement models. Functions related to growth, feeding, and energy were all taken from the literature and parameters were based on Danish data records. The biological model for reproduction used to calculate probability of pregnancy in the MDP was a detailed model of the estrus cycle. Some of the parameters were taken from the literature whereas the rest were management decisions (insemination period and time of pregnancy test). Because of the daily time steps in the MDP, the estrus cycle could be modeled much better than for models with time steps of $1 \mathrm{mo}$, which not is a good representation of the 3 -wk estrus cycle.

The model obviously also lacked some features that may be useful in a herd management system. The model did not consider seasonal effects (van Arendonk, 1986; Delorenzo et al., 1992), which may be important to include if there are differences in lactation curves and prices over the year.

Decisions related to insemination and the treatment of a disease were not included explicitly in the model. This was beyond the scope of this paper. However, note that because the model considered daily stages, real-time decisions for insemination and treatment can be included by adding relevant state variables to the state space of the HMDP. The new state variables must relate to the biosensors in the farm. For example, online measurements of electric conductivity or the enzyme lactate dehydrogenase can be used to predict mastitis (Norberg et al., 2004; Friggens et al., 2007) and progesterone measurements can be used to predict the best time of insemination (Nebel, 1988). The number of new state variables has to be limited, though, because of dimensionality problems for MDP models that state that the number of states grows exponentially with the number of state variables. For example, including 2 new state variables with 2 levels in the HMDP will make the total number of states grow from 3,011,884 to approximately $12,047,536(2 \times 2 \times 3,011,884)$. Dimensionality is a critical problem in integrating new state variables and advanced ways to circumvent the problem are relevant.

Because the HMDP framework focused on the individual cow, herd constraints were not included in the model. For example, a limited number of heifers available may delay the replacement of a cow. However, in this case the economic values of the cows found using the HMDP can be used to rank the cows in the herd.

The dynamic model described in this paper is a step in the direction of an HMDP model that can be integrated into a herd management system. Future research includes integrating decisions related to insemination and diseases, which include methods that take into account the dimensionality problem.

\section{ACKNOWLEDGMENTS}

This study was funded by a grant financed by the Directorate for Food, Fisheries and Agri Business (Copenhagen, Denmark), Lattec I/S (Hillerød, Denmark), the Danish Cattle Association (Aarhus, Denmark), and the Faculty of Agricultural Sciences (Tjele, Denmark).

\section{REFERENCES}

Bar, D., L. W. Tauer, G. Bennett, R. N. Gonzalez, J. A. Hertl, Y. H. Schukken, H. F. Schulte, F. L. Welcome, and Y. T. Grohn. 2008. The cost of generic clinical mastitis in dairy cows as estimated by using dynamic programming. J. Dairy Sci. 91:2205-2214.

Bar, D., L. W. Tauer, G. Bennett, R. N. Gonzalez, J. A. Hertl, Y. H. Schukken, H. F. Schulte, F. L. Welcome, and Y. T. Grohn. 2009. Use of a dynamic programming model to estimate the value of clinical mastitis treatment and prevention options utilized by dairy producers. Agric. Syst. 99:6-12.

Bascom, S. S., and A. J. Young. 1998. A summary of the reasons why farmers cull cows. J. Dairy Sci. 81:2299-2305.

Beaudeau, F., V. Ducrocq, C. Fourichon, and H. Seegers. 1995. Effect of disease on length of productive life of French Holstein dairy cows assessed by survival analysis. J. Dairy Sci. 78:103-117.

Dechow, C. D., and R. Goodling. 2008. Mortality, culling by sixty days in milk, and production profiles in high-and low-survival Pennsylvania herds. J. Dairy Sci. 91:4630-4639.

Delorenzo, M. A., T. H. Spreen, G. R. Bryan, D. K. Beede, and J. A. M. van Arendonk. 1992. Optimizing model: Insemination, replacement, seasonal production, and cash flow. J. Dairy Sci. $75: 885-896$.

Friggens, N. C., M. G. G. Chagunda, M. Bjerring, C. Ridder, S. Højsgaard, and T. Larsen. 2007. Estimating degree of mastitis from time-series measurements in milk: A test of a model based on lactate dehydrogenase measurements. J. Dairy Sci. 90:54155427.

Friggens, N. C., K. L. Ingvartsen, and G. C. Emmans. 2004. Prediction of body lipid change in pregnancy and lactation. J. Dairy Sci. 87:988-1000.

Giaever, H. 1966. Optimal dairy cow replacement policies. PhD thesis. University of Michigan, Ann Arbor.

Groenendaal, H., D. T. Galligan, and H. A. Mulder. 2004. An economic spreadsheet model to determine optimal breeding and replacement decisions for dairy cattle. J. Dairy Sci. 87:2146-2157.

Hastie, T. 1993. Generalized Additive Models. Chapman \& Hall, Inc., New York, NY.

Heikkila, A. M., J. I. Nousiainen, and L. Jauhiainen. 2008. Optimal replacement policy and economic value of dairy cows with diverse health status and production capacity. J. Dairy Sci. 91:23422352.

Houben, E. H., R. Huirne, A. Dijkhuizen, and A. Kristensen. 1994. Optimal replacement of mastitis cows determined by a hierarchic Markov process. J. Dairy Sci. 77:2975-2993.

Jenkins, K. B., and A. N. Halter. 1963. A multistage stochastic replacement decision model: Application to replacement of dairy 
cows. Page 67 in Technical bulletin: Agric. Exp. Sta. Oregon State University, Corvallis.

Jørgensen, E. 2009. Technical report (internal): A semi-Markov chain model for the reproduction cycle. Department of Genetics and Biotechnology, Aarhus University, Aarhus, Denmark.

Kennedy, J. O. S., and A. W. Stott. 1993. An adaptive decision-making aid for dairy cow replacement. Agric. Syst. 42:25-39.

Korver, S., and J. A. M. van Arendonk. 1988. Modelling of Livestock Production Systems. Kluwer Academic Publishers, Dordrecht, the Netherlands.

Kristensen, A. 1987. Optimal replacement and ranking of dairy cows determined by a hierarchical Markov process. Livest. Prod. Sci. $16: 131-144$.

Kristensen, A. 1989. Optimal replacement and ranking of dairy cows under milk quotas. Acta Agric. Scand. 39:311-318.

Kristensen, A. 2003. A general software system for Markov decision processes in herd management applications. Comp. Electric. Agric. 38:199-215.

Kristensen, A. R., and E. Jørgensen. 2000. Multi-level hierarchic Markov processes as a framework for herd management support. Ann. Oper. Res. 94:69-89.

Kristensen, E., S. Østergaard, M. A. Krogh, and C. Enevoldsen. 2008. Technical indicators of financial performance in the dairy herd. J. Dairy Sci. 91:620-631.

Monti, G., B. A. Tenhagen, and W. Heuwieser. 1999. Culling policies in dairy herds. A review. Zentralbl. Veterinarmed. A 46:1-11.

National Research Council. 2000. Page 232 in Nutrient Requirements of Beef Cattle. National Research Council, Washington, D.C.

Nebel, R. L. 1988. On-farm milk progesterone tests. J. Dairy Sci. 71:1682-1690

Nielsen, L. 2008. Technical report: Embedding a state space model into a Markov decision process. Department of Genetics and Biotechnology, Aarhus University, Aarhus, Denmark.

Norberg, E., H. Hogeveen, I. Korsgaard, N. Friggens, K. Sloth, and P. Lovendahl. 2004. Electrical conductivity of milk: Ability to predict mastitis status. J. Dairy Sci. 87:1099-1107.

Østergaard, S., M. Chagunda, N. Friggens, T. W. Bennedsgaard, and I. C. Klaas. 2005. A stochastic model simulating pathogen-specific mastitis control in a dairy herd. J. Dairy Sci. 88:4243-4257.

Pinheiro, J., D. Bates, S. DebRoy, and S. Sarkar. 2006. nlme: Linear and Nonlinear Mixed Effects Models. R package version 3.1-78. http://cran.r-project.org/web/packages/nlme/index.html

Rajala-Schultz, P. J., Y. Grohn, and H. Allore. 2000. Optimizing breeding decisions for Finnish dairy herds. Acta Vet. Scand. 41:199-212.

Sargent, R. G. 2008. Verification and validation of simulation models. Pages 157-169 in Proceedings of the 40th Conference on Winter Simulation. Miami, FL.

Sørensen, J. T. 1990. Validation of livestock herd simulation models: A review. Livest. Prod. Sci. 26:79-90.

Stott, A. 1994. The economic advantage of longevity in the dairy cow. J. Agric. Econ. 45:113-122.

R Development Core Team. 2008. R: A Language and Environment for Statistical Computing. R Foundation for Statistical Computing, Vienna, Austria.

van Arendonk, J. A. M. 1986. Studies on the replacement policies in dairy cattle. IV. Influence of seasonal variation in performance and prices. Livest. Prod. Sci. 14:15-28.

Vargas, B., M. Herrero, and J. van Arendonk. 2001. Interactions between optimal replacement policies and feeding strategies in dairy herds. Livest. Prod. Sci. 69:17-31.

Verstegen, J. A. A. M., R. B. M. Huirne, A. A. Dijkhuizen, and J. P. C. Kleijnen. 1995. Economic value of management information systems in agriculture: A review of evaluation approaches. Comp. and Electric. in Agric. 13:273-288

Vestergaard, M., N. T. Madsen, H. B. Bligaard, L. Bredahl, P. T. Rasmussen, and H. R. Andersen. 2007. Consequences of two or four months of finishing feeding of culled dry dairy cows on carcass characteristics and technological and sensory meat quality. Meat Sci. 13:635-643.
West, M., and J. Harrison. 1997. Bayesian Forecasting and Dynamic Models. 2nd ed. Springer-Verlag, New York, NY.

Wilmink, J. B. M. 1987. Adjustment of test-day milk, fat and protein yield for age, season and stage of lactation. Livest. Prod. Sci. 16:335-348.

Wood, P. D. P. 1967. Algebraic model of the lactation curve in cattle. Nature 216:164-165.

Yalcin, C., and A. Stott. 2000. Dynamic programming to investigate financial impacts of mastitis control decisions in milk production systems. J. Dairy Res. 67:515-528.

\section{APPENDIX: PARAMETERS OF BIOLOGICAL FUNCTIONS}

An overview of the parameters in the biological functions is given.

\section{Functions Related to Milk Yield}

The model for daily milk yield is given in equation [1]. The curves were based on milk recordings from a robot milking system (DeLaval, Tumba, Sweden) at the Danish Cattle Research Centre in Denmark. Daily milk yield for each cow $\left(M_{t, j}\right)$ was calculated as the sum of the milk recordings from each day for each cow and each lactation.

Because of occasional recording failures (e.g., robot malfunctioning or severe disease periods), a preliminary data analysis was performed using $\log \left(M_{t, j}\right)$ as dependent variable and omitting the autoregressive term $X_{t, j}$ in equation [1]. The recording failures resulted in clearly recognizable patterns in the residuals. Thus, based on inspection of the residuals, 7 lactation periods and, in addition, some daily recordings with very low residuals, were omitted from the data set. The final data analysis included a total of 189 cows with an average of 2.9 lactation periods for each cow. Parity 1 cows were included only if they had a second lactation.

The average curve was estimated using a spline function with 6 degrees of freedom. A B-spline basis (see Hastie, 1993) for the polynomial spline was used, which was calculated using the $\mathrm{bs}()$ function in the splines package in $\mathrm{R}$ ( $\mathrm{R}$ Development Core Team, 2008). Parameters were estimated using the lme function in the nlme package in $\mathrm{R}$ (Pinheiro et al., 2006). The effect of cow was specified as a random effect and the autoregressive and residual terms specified using the corExp correlation structure with a nugget effect.

Estimation based on the model in equation [1] often results in difficulties with parameter estimation. If $\rho \sim$ $1, X_{t, j}$ becomes almost constant and cannot be distinguished from the permanent effect of animal, A. Especially in cases with relatively short time-series, this is a problem. To diminish this problem, a 2-step estimation procedure was used. In the first step the model param- 


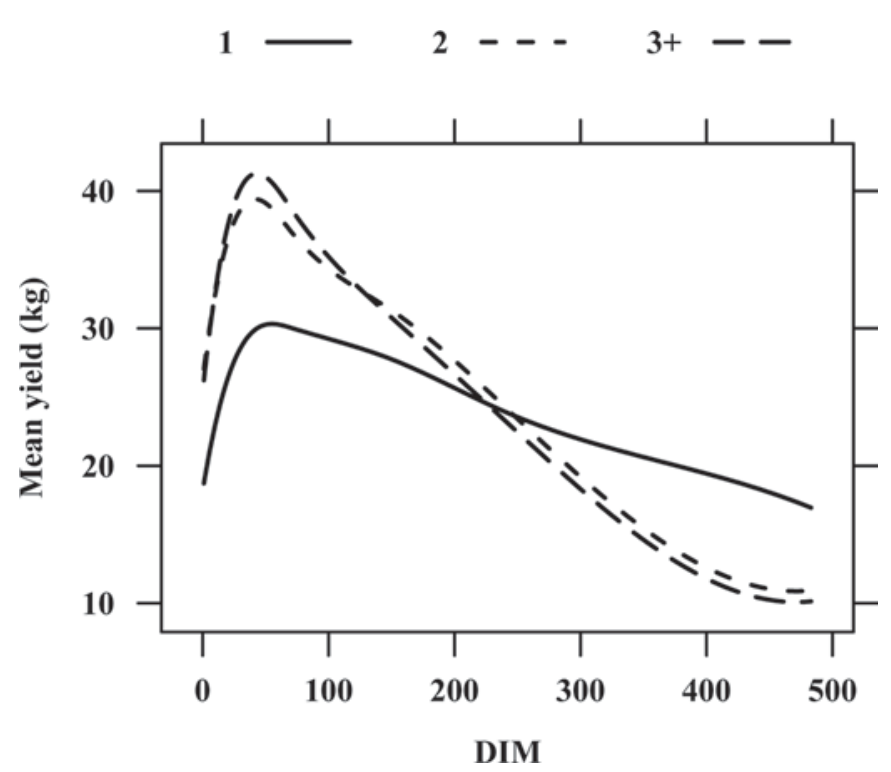

Figure 8. Average milk yield of the herd for different lactations $(1,2$, and $3+)$.

eters were estimated without restrictions. In the second step the variance parameters were recalculated based on an assumption that $99 \%$ of the covariance between milk yields $200 \mathrm{~d}$ apart was a result of the variance component for the production potential $\left(\sigma_{A}^{2}\right)$. This corresponds to an upper limit on the autocorrelation coefficient $\rho$ of $\sim 0.98$. In a second call to the lme() function, the revised parameters for the correlation structure was assumed fixed (i.e., only $\sigma_{A}^{2}$ was estimated).

The estimated values of the variance parameters of the SSM are given in Table 2. A plot of average daily milk yield at herd-level $\left(\mu_{t, j}\right)$ is given in Figure 8. The daily milk of a cow with expected production potential and local production effect $m$ (see equation [5]) can be calculated using equation [7].

\section{Parameters and Functions Related to Growth and Age}

The total BW (excluding the weight of the fetus) of the cow is based on a combination of 2 curves. The first is a Gompertz curve used to describe the BW of the animal corrected to a BCS of 3 (5-point scale) and excluding any weight of the fetus (stdBW):

$\operatorname{stdBW}($ lac,DIM $)=a \exp \left\{-b \exp \left[-c\left(\operatorname{age}_{p}+\mathrm{DIM}\right)\right]\right\}$,

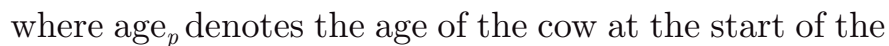
lactation (Kristensen et al., 2008).

The second is a BCS function BCS(DIM,DOC) taken from Friggens et al. (2004) that is dependent on DIM

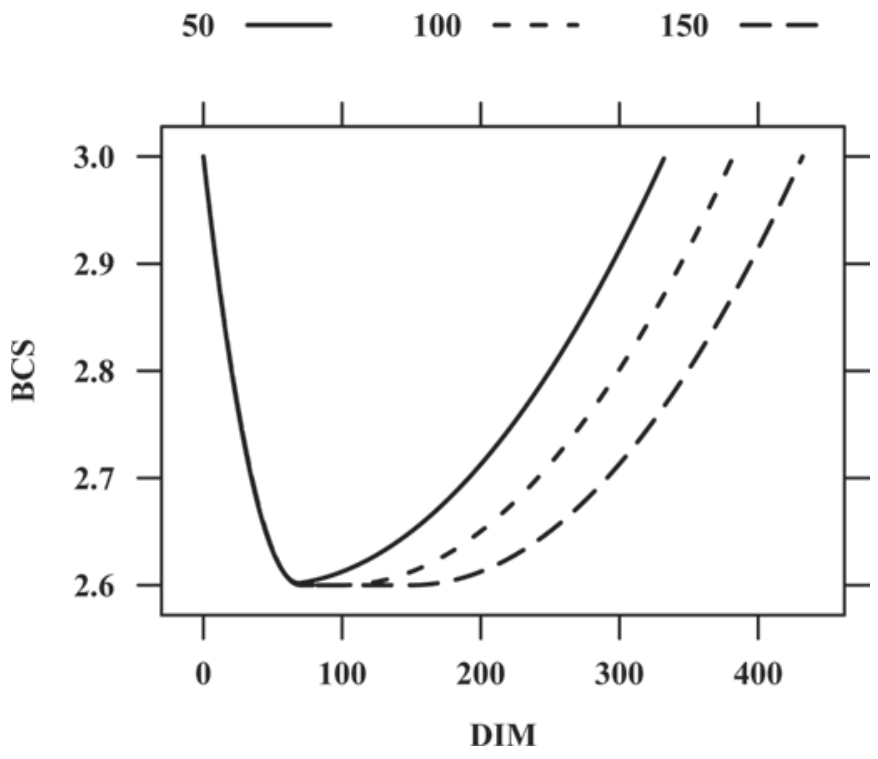

Figure 9. Body condition score during lactation for different days of conception (50,100, and 150).

and day of conception (DOC). Here we assume that the $\mathrm{BCS}$ at calving is $\mathrm{BCS}(0, \mathrm{DOC})=3$; at time $\mathrm{t}=70$ the daily BCS change stops being negative, with a BCS of $\mathrm{BCS}(\mathrm{T}, \mathrm{DOC})=2.6$. A gestation length of $282 \mathrm{~d}$ is assumed and the BCS at next calving is assumed to be $\mathrm{BCS}(\mathrm{DOC}+282, \mathrm{DOC})=3$. Finally, a daily BCS loss of at most 0.0324 is assumed.

A plot of the BCS is given in Figure 9. Figure 10 shows the total BW of the cow for different lactations.

The weight of the fetus (fetusBW) is given as (National Research Council, 2000)

$$
\begin{gathered}
\text { fetusBW(DOC,DIM })= \\
a b \exp [-c \times(282+\text { DOC }- \text { DIM })] .
\end{gathered}
$$

An overview of the values of the parameters $a, b$, and $c$ used in the functions stdBW and fetusBW is given in Table 3.

Table 2. Estimated variance parameters ${ }^{1}$ of the state space model for the yield

\begin{tabular}{lcccc}
\hline Lactation (n) & $\sigma_{A_{j}}$ & $\sigma_{X_{j}}$ & $\rho_{j}$ & $\sigma_{\nu_{j}}$ \\
\hline 1 & 4.99 & 5.63 & 0.98 & 6.36 \\
2 & 4.63 & 4.74 & 0.98 & 6.36 \\
$3+$ & 4.08 & 4.74 & 0.98 & 6.36 \\
\hline
\end{tabular}

$\sigma_{A_{j}}=\mathrm{SD}$ for the production potential $A$ in lactation $j ; \sigma_{X_{j}}=\mathrm{SD}$ for the local production effect $X ; \rho_{j}=$ correlation coefficient of $X ; \sigma_{\nu_{j}}=$ $\mathrm{SD}$ for the measurement error. 


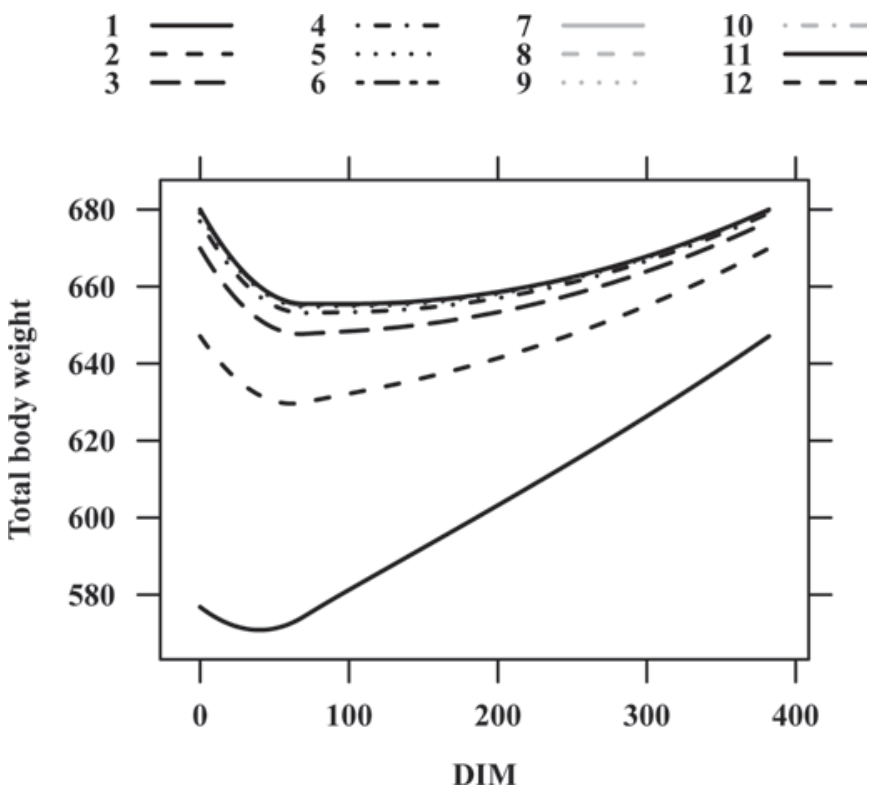

Figure 10. Body weight $(\mathrm{kg})$ for different lactations (day of conception at d 100; fetus weight not included).

\section{Functions Related to Energy and Feeding}

The daily feed intake of the cow is measured in net energy of Scandinavian Feed Units (SFU). The total amount of energy is the sum of energy needed for maintenance (eMain), fetus growth (eFetus), milk yield (eECM), standardized BW gain (eBWG)

$$
\begin{gathered}
\text { eMain }(\text { lac,DIM })=a\left(\frac{\operatorname{stdBW}(\text { lac,DIM })}{b}+c\right) \\
\operatorname{eFetus}(\text { DIM,DOC })=a \times \text { fetusBW }(\text { DOC,DIM }) \\
\operatorname{eECM}(\text { lac,DIM,m })=a \times \text { yield } 2 \mathrm{ECM}(\mathrm{M})
\end{gathered}
$$

$\operatorname{eBWG}(\operatorname{lac}, \mathrm{DIM})=a[\operatorname{stdBW}(\operatorname{lac}, \mathrm{DIM}+1)-\operatorname{stdBW}($ lac, DIM $)]$,

and BCS change (eBCS)

$\operatorname{eBCS}(\mathrm{DIM}, \mathrm{DOC})=\left\{\begin{array}{ll}\mathrm{dBCS} \times[a \times \mathrm{BCS}(\mathrm{DIM}, \mathrm{DOC})+b] & \mathrm{dBCS}>0 \\ \mathrm{dBCS} \times[c \times \mathrm{BCS}(\mathrm{DIM}, \mathrm{DOC})+d] & \text { otherwise }\end{array}\right.$,

where dBCS denotes the daily change in BCS. The total energy requirements (eTotal) now become

$$
\begin{aligned}
& \text { eTotal }(\text { lac,DIM,DOC, } m) \\
& =\operatorname{eMain}(\text { lac,DIM })+\text { eFetus }(\text { DIM,DOC }) \\
& +\operatorname{eECM}(\text { lac,DIM }, m)+\operatorname{eBWG}(\text { lac,DIM })+\operatorname{eBCS}(\text { DIM,DOC })
\end{aligned}
$$

An overview of the parameters used in each function is given in Table 3.

\section{Functions Related to Reproduction}

A reproduction model described in Jørgensen (2009) has been used to calculate the probability of a positive pregnancy test given an insemination $40 \mathrm{~d}$ before [function prPosPregT(DIM)]. The probability is shown in Figure 11. In the calculations we use an average length of the estrus cycle of $21 \mathrm{~d}$ with variance 2 . Probability of insemination given estrus was set to $50 \%$, probability of pregnancy given insemination was $45 \%$. Inseminations were continued until $250 \mathrm{~d}$ after calving and not started before d 35 .

\section{Functions Related to IC}

The probability of IC is based on a piecewise linear function for the hazard rate $(h)$ or intensity for IC. In the model these hazard rates are used to calculate the

Table 3. Overview over parameters ${ }^{1}$ related to growth, energy, and feeding functions ${ }^{2}$

\begin{tabular}{lcccc}
\hline Item $^{3}$ & \multicolumn{1}{c}{$a$} & $b$ & $c$ & $d$ \\
\hline stdBW(lac,DIM) & 680 & 2.5483 & 0.00314 & - \\
BW(lac,DIM,DOC) & 0.09 & - & - & - \\
fetusBW(DOC,DIM) & 680 & 0.1133 & 0.02 & - \\
eMain(lac,DIM) & 1.1 & 200 & 1.5 & - \\
eFetus(DIM,DOC) & 0.03647 & - & - & - \\
eECM(lac,DIM, $\hat{A}, \hat{X})$ & 0.4 & - & - & - \\
eBWG(lac,DIM) & 4 & - & - & - \\
eBCS(DIM,DOC) & 0.4857 & 1.3857 & 0.4714 & 1.0857 \\
\hline
\end{tabular}

${ }^{1}$ The parameters $a, b, c$, and $d$ for each formula. Parameter not present is indicated with a dash.

${ }^{2}$ The input parameters in the functions are lactation (lac), day of conception (DOC), the expected production potential $(\hat{A})$, and the expected local production effect $(\hat{X})$.

${ }^{3}$ stdBW $=$ the standardized $\mathrm{BW}$ (BCS $=3$ and excluding the weight of the fetus) of the cow; fetusBW = the weight of the fetus; eMain $=$ energy needed for maintenance; eFetus $=$ energy needed for fetus growth; eECM = energy needed for milk yield; eBWG = energy needed for standardized BW gain; eBCS = energy needed for BCS change. For eTotal (the sum of eMain, eFetus, eECM, eBWG, and eBCS), see equation [8]. 


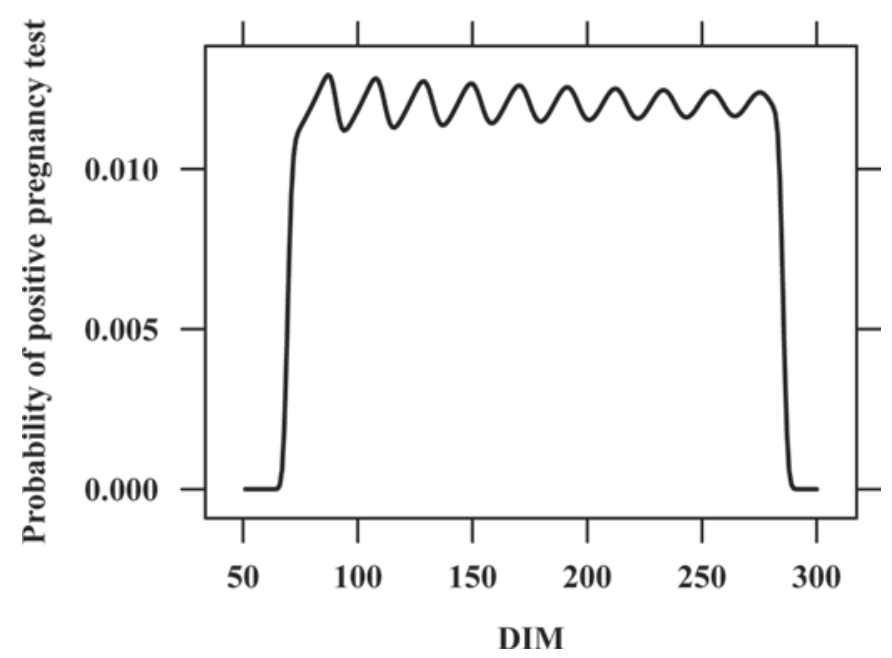

Figure 11. Probability of positive pregnancy test.

conditional probability that the cow is culled within the next day given that it still is alive. To ease the interpretation of hazard rates, they are transformed to $p=$ $1-\exp (-350 \times h)$; that is, the percentages of animals culled in a 350-d period, if the hazard rate was kept constant at $h$. In the model, for instance in lactation 1 , the logarithm to the hazard rate falls linearly from calving $(p=0.3)$ to the end of the voluntary waiting period $(p=0.1)$, as illustrated by the solid line in Figure 12 . This corresponds to a reduction in $h$ from 0.0010 to 0.0003 . Then the hazard rate is constant until either a positive pregnancy test or it is decided to stop mating. In this paper the hazard rate in both cases corresponds to $p=0.2$. However, we may have different hazard rates in the 2 cases. In the final period from drying off to calving, the risk of IC does not depend on the length of the period and is expressed as the probability in the whole period $(p=0.1)$. The same patterns are used for the other lactations but with an increasing offset to indicate increasing IC rates. The daily and accumulated probability of IC is shown in Figure 12.

\section{Discretizing the SSM}

Considering the SSM for the milk yield the density of $\left[\left(\hat{A}_{t, j}, \hat{X}_{t, j}\right) \mid\left(\hat{A}_{t-1, j}, \hat{X}_{t-1, j}\right)\right]$ can be calculated using standard formulas for a linear transformation of the multivariate normal distribution (see Nielsen, 2008):

$$
\left[\left(\hat{A}_{t, j}, \hat{X}_{t, j}\right) \mid\left(\hat{A}_{t-1, j}, \hat{X}_{t-1, j}\right)\right] \sim N\left[\left(\hat{A}_{t-1, j}, \rho \hat{X}_{t-1, j}\right)^{\prime}, Q_{t} \mathbf{B}_{t} \mathbf{B}_{t}^{\prime}\right],
$$

where $\quad \mathbf{B}_{t}=\mathbf{R}_{t, j} \mathbf{F} Q_{t, j}^{-1}, \quad \mathbf{R}_{t, j}=\mathbf{G C}_{t-1, j} \mathbf{G}^{\prime}+\mathbf{W}, \quad$ and $Q_{t, j}=\mathbf{F}^{\prime} \mathbf{R}_{t, j} \mathbf{F}+\sigma_{\nu}^{2}$. Matrices $\mathbf{F}, \mathbf{G}$, and $\mathbf{W}$ are defined in equation [3].

To embed the SSM in the HMDP, the 2-dimensional space of $(\hat{A}, \hat{X})$ must be discretized into the set $\Phi_{(\hat{A}, \hat{X})}$. The approximation is made using the nonuniform discretization illustrated in Figure 13, which partitions the
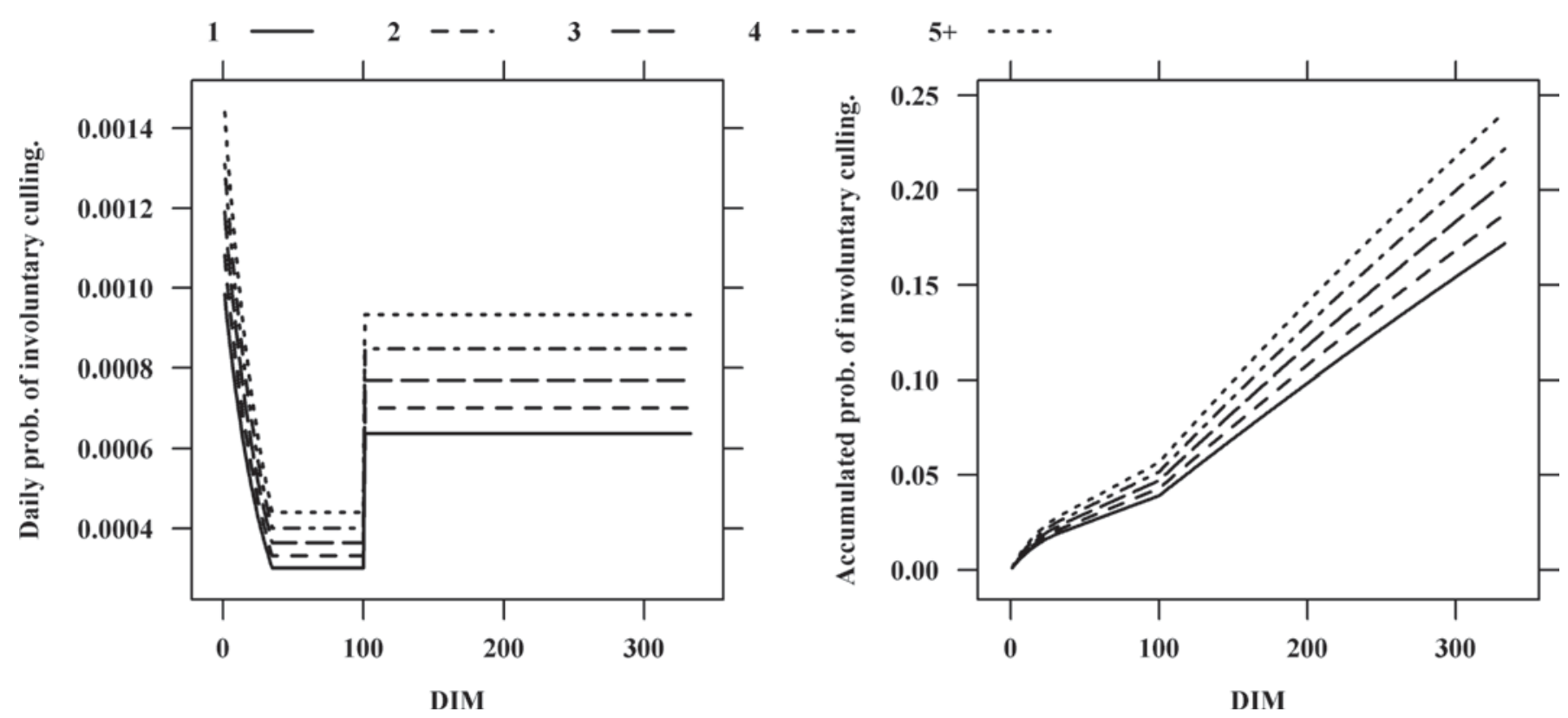

Figure 12. Daily probability of involuntary culling (left) and accumulated probability of involuntary culling (right) of a cow for different lactations (day of conception at d 100). 
space into squares of various sizes. Each square represents an element in $\Phi_{(\hat{A}, \hat{X})}$. For a detailed overview of how to make this discretization, see Nielsen (2008).

Let $(\hat{A}, \hat{X})_{t}$ and $(\hat{A}, \hat{X})_{t-1}$ denote the center point in squares $\theta_{2}$ and $\theta_{1}$, respectively. To calculate the probability $\operatorname{prM}\left[(\hat{A}, \hat{X})_{t},(\hat{A}, \hat{X})_{t-1}, t\right]$ of a transition from square $\theta_{1}$ to $\theta_{2}$ in Figure 13 we use the following formula:

$$
\operatorname{prM}\left[(\hat{A}, \hat{X})_{t},(\hat{A}, \hat{X})_{t-1}, t\right]=\int_{(\hat{A}, \hat{X}) \in \theta_{2}} p(\hat{A}, \hat{X}) d(\hat{A}, \hat{X}),
$$

where $p(\cdot)$ denotes the multivariate normal density of equation [9].

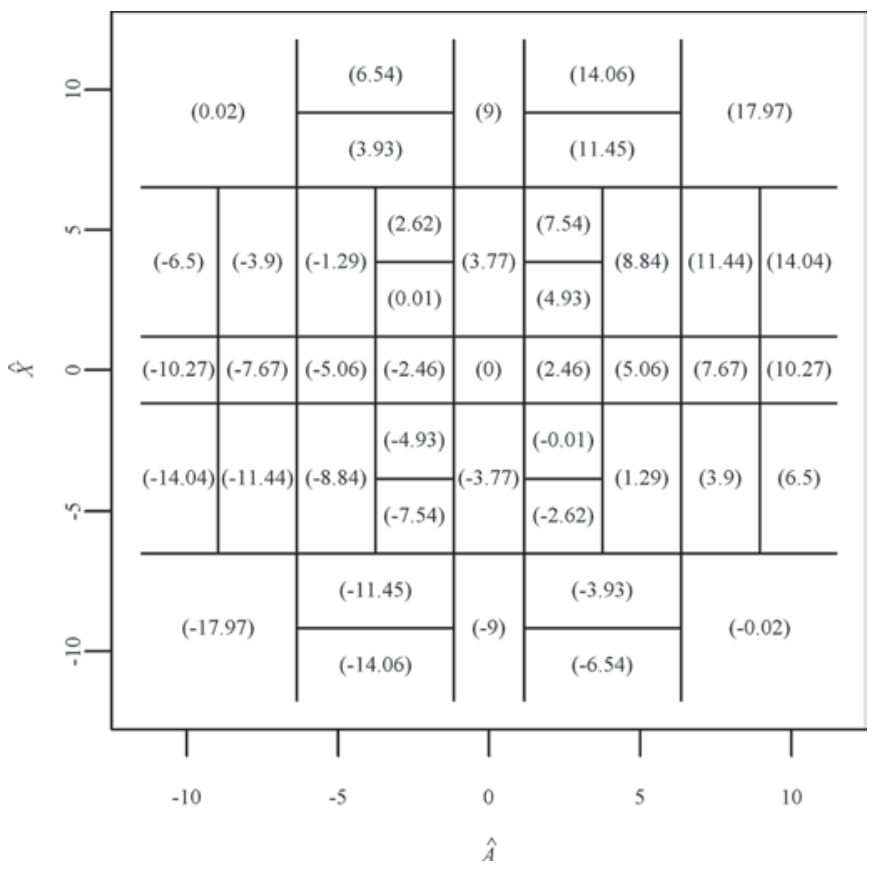

Figure 13. Nonuniform discretization of the expected production potential $(\hat{A})$ and the expected local production effect $(\hat{X})$ into the set $\Phi_{(\hat{A}, \hat{X})}$. Numbers in parentheses are the expected residual milk yield. 